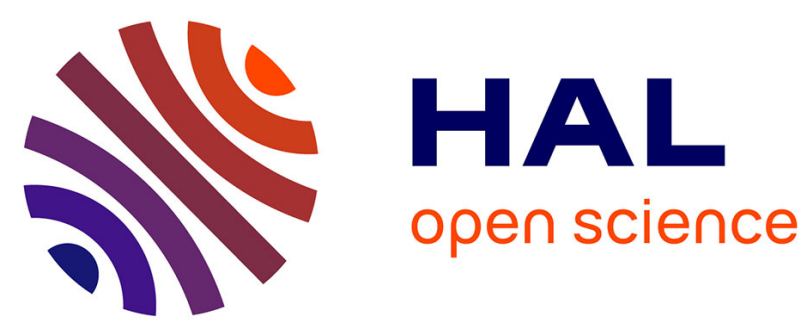

\title{
Mars Organic Molecule Analyzer (MOMA) Flight Analog Activities in Preparation for ExoMars Surface Operations
}

\author{
William Brinckerhoff, Fred Goesmann, François Raulin, Cyril Szopa
}

\section{- To cite this version:}

William Brinckerhoff, Fred Goesmann, François Raulin, Cyril Szopa. Mars Organic Molecule Analyzer (MOMA) Flight Analog Activities in Preparation for ExoMars Surface Operations. European Planetary Science Congress. EPSC 2021, Sep 2021, Virtual Meeting, United States. 10.5194/epsc2021-470 . insu-03323060

\section{HAL Id: insu-03323060 \\ https://hal-insu.archives-ouvertes.fr/insu-03323060}

Submitted on 20 Aug 2021

HAL is a multi-disciplinary open access archive for the deposit and dissemination of scientific research documents, whether they are published or not. The documents may come from teaching and research institutions in France or abroad, or from public or private research centers.
L'archive ouverte pluridisciplinaire HAL, est destinée au dépôt et à la diffusion de documents scientifiques de niveau recherche, publiés ou non, émanant des établissements d'enseignement et de recherche français ou étrangers, des laboratoires publics ou privés.

\section{(c)(1)}

Distributed under a Creative Commons Attribution| 4.0 International License 


\title{
Mars Organic Molecule Analyzer (MOMA) Flight Analog Activities in Preparation for ExoMars Surface Operations
}

\author{
William Brinckerhoff ${ }^{1}$, Fred Goesmann ${ }^{2}$, Francois Raulin ${ }^{3}$, and Cyril Szopa ${ }^{4}$ \\ ${ }^{1}$ NASA Goddard Space Flight Center, Greenbelt, MD 20771 USA (william.b.brinckerhoff@nasa.gov) \\ ${ }^{2}$ Max-Planck-Institut für Sonnensystemforschung, Justus-von-Liebig-Weg 3, 37077 Göttingen, Germany \\ ${ }^{3}$ Laboratoire Interuniversitaire des Systèmes Atmosphériques (LISA), 61, Avenue du Général de Gaulle 94010 Créteil Cedex, \\ France \\ ${ }^{4}$ Laboratoire Atmosphères, Observations Spatiales (LATMOS), 11 Boulevard d'Alembert 78280 Guyancourt, France
}

The Mars Organic Molecule Analyzer (MOMA) investigation is part of the scientific payload of the ExoMars rover Rosalind Franklin that will arrive at Oxia Planum on Mars in June, 2023. MOMA is a dual-source (laser desorption, LD, and gas chromatography, GC) mass spectrometer (MS) that will analyze the organic chemical composition of collected samples in support of the ExoMars objective to seek the signs of life in the near subsurface (up to two meters depth) of Mars [1]. The dualsource design allows MOMA to provide broadly sensitive detection of organics over a range of molecular weights and volatilities, as well as probe structural patterns of martian molecules such as enantiomeric ratios or chain length biases in aliphatic moieties.

A number of important MOMA activities are being carried out in preparation for ExoMars launch in 2022 and science mission beginning in mid-2023. These activities will optimize the readiness of all relevant MOMA hardware (both flight and reference) and of the full team for surface operation. These include development and certification of the MOMA Testbed Model that will serve as a flight reference instrument, analysis of Mars analog samples with MOMA laboratory capabilities, and preparation for data analysis, decision making, and risk management during the rover mission.

The Testbed Model is a ground-based duplicate of the Flight Model (FM), serving a dual role as (i) an engineering reference for verification and validation of flight procedures and for diagnosing flight performance (including any off-nominal situations); and (ii) a science reference with direct traceability to the sensitivity, background, and other characteristics of the FM, with the potential to carry out selected analog studies for scientific interpretation of MOMA flight data.

The Testbed must always retain full flight fidelity and be available on tactical (sol-to-sol) timeframes (more-or-less continuously) to serve its engineering reference functions. It can thus be used to test modified operational software scripts to be run subsequently on the FM. There is additionally a strong bias to maintaining the Testbed in a state of low chemical background and overall performance, comparable to the FM, to retain its ability to rule-in/out any detections of lowconcentration organic compounds on Mars. As such the bar for introduction of complex Mars analog samples into the Testbed must be set very high, and must be managed responsibly over the mission duration. Only those analyses requiring the full fidelity of the FM, under simulated Mars ambient conditions, for interpretation of critical data products would normally be considered for significant 
scientific use of the Testbed.

A variety of sample studies are conducted in MOMA team laboratories, including use of the Engineering Test Unit (ETU) which lacks the Mars thermal-vacuum (Mars ambient pressure) environmental facility of the Testbed but is otherwise a high-fidelity scientific performance reference of MOMA used for analysis of Mars analog materials. Some of the ongoing and planned ETU-based test campaigns include: (i) development of detailed protocols for collection of LD mass spectra, comparing point-by-point and multi-pulse averaging modes [2] and use of isolation and tandem mass spectrometry, or MS/MS, capabilities to inform chemical assignments; (ii) characterization of the dependence on geochemical (e.g. sample matrix) and environmental factors during analysis of organic compounds using derivatization and GCMS, to include a recently-upgraded GC ETU supporting flight-like column and gas processing line temperatures; and (iii) participation in ExoMars collaborative analysis campaigns, using additional shared analogs including designated "mission samples" (matching projected compositions at Oxia Planum), alongside other payload investigations such as the MicrOmega and Raman spectrometers of the Analytical Laboratory Drawer (ALD).

In addition to developing and testing flight-like experimental resources, the MOMA team is actively involved in preparations for ExoMars operations across the spectrum of planning and training for telemetry management, science analysis, and decision-making roles and processes. Team members are participating in rover simulation and software tool training hosted by the Rover Operations Control Center (ROCC) managed by ALTEC SpA, Turin, Italy. As a nominal "end-point" analysis of collected samples during each Experiment Cycle and Vertical Survey campaign, MOMA has significant interest in collaborating fully with all rover scientific payload teams to develop target interpretations and prioritizations based on remote, contact, and collected-sample analyses. As such mission simulations merging and synchronizing strategic and tactical processes of increasing fidelity are critical.

Within the context and structure of these rover-level interactions and flows, the MOMA team additionally is establishing a set of scenario- and data-based evaluation metrics and decision-making procedures to aid in efficient and robust science operations in line with overall MOMA analytical objectives. One line of science scenario preparation is understanding what aspects of sample characteristics and preliminary mineralogy, as determined by other rover instruments, will be used to set initial MOMA parameters for LDMS or GCMS runs. Such settings are empirically based on laboratory data as well as rover mission data up to that point. Any follow-up analyses by MOMA on the same sample, such as selection of a particular type of chemical derivatization and GC column, would similarly depend on advance understanding based on analog and flight data.

The need for decision making preparation applies to non-optimal, unexpected, and/or anomalous situations as well as nominal operations. Given the few-hour turnaround for tactical decisions, to avoid the potential for loss of a sol MOMA is developing policies and procedures to determine the nature of the situation, and work in conjunction with the rover team to assess options, and to convey, through the MOMA PI and operations team, a recommended path forward, based on prior simulations and flight experience. Examples of such cases may include finding that less (or more) energy is available for a given MOMA run than planned, or more concerning, receiving anomalous data products (e.g., noise) or alarmed housekeeping values.

The above activities, carried out across the MOMA team, provide critical inputs to the planned Operational Readiness Reviews in mid-2022 and allow the MOMA investigation and the ExoMars mission to meet its higher-level goals.

- Goesmann F et al. (2017) Astrobiology 17:655-685. https://doi.org/10.1089/ast.2016.1551

- Siljeström S et al. (2021) Astrobiology. https://doi.org/10.1089/ast.2020.2368 